\title{
FINITE DENSITY QCD SUM RULES FOR NUCLEONS
}

\author{
E. G. Drukarev, *
}

November 7, 2018

\begin{abstract}
It is shown how the QCD sum rules can be applied for the investigation of the density dependence of the nucleon parameters. These characteristics can be expressed through the expectation values of QCD operators in nuclear matter. In certain approximations the expectation values are related to the observables. First applications of the approach reproduced some of the basic features of nuclear physics, providing also a new knowledge. The program of the future work is presented. The difficulties of the approach are discussed.
\end{abstract}

\section{Contents}

1. Motivation

2. QCD sum rules in vacuum

2.1. Dispersion relations

2.2. Sum rules

3. QCD sum rules in nuclear matter

3.1. The problems

3.2. Lowest order OPE terms

*present address: PNPI, Gatchina, St. Petersburg 188300, Russia, e-mail: drukarev@thd.pnpi.spb.ru 
3.3. Gas approximation. The role of $\pi N$ sigma-term in nuclear physics

3.4. Beyond the gas approximation. A possible saturation mechanism

3.5. Higher order OPE terms

3.6. New knowledge

3.7. Self-consistent scenario

3.8. A sub-plot: Goldstone pions never condense

\section{Summary}

The lecture is not addressed to the experts. The aim of the talk is rather to attract attention of the researchers who just started to study the subject. The third section is based on the results obtained in collaboration with M. G. Ryskin, V. A. Sadovnikova and E. M. Levin.

\section{Motivation}

The theory of nuclear matter leaves some room for the improvement. This concerns the low densities, i.e. those close to the saturation value as well as the higher densities.

Since the pioneering paper of Walecka [1] the partially successful Schrödinger phenomenology was succeeded by still more successful Dirac phenomenology. A nucleon in nuclear matter is treated as moving in superposition of the scalar and vector fields which are of several hundreds $\mathrm{MeV}$. In the meson-exchange picture of nucleon interactions these fields originate from the exchange by $\sigma$ and $\omega$ mesons. This model is known as quantum hadrodynamics (QHD). It is quite successful in describing most of the properties of nucleons in both nuclear matter and finite nuclei [2, 3]. However the model is not fundamentally complete. The weak points of QHD were reviewed by Negele 4 and by Sliv et al [5]. Here I mention that " $\sigma$-meson" is rather an effective way of describing the scalar interactions. Also the masses $m_{\sigma, \omega}$ of $\sigma$ - and $\omega$-mesons are so large that the scalar and vector interactions take place at the distances where the nucleons cannot be treated as the point particles any more. Finally, the coupling constants $g_{\sigma}$ and $g_{\omega}$ are the free parameters of QHD. They are chosen usually to fit the saturation properties.

Thus it would be desirable to develop the approach which has the attractive features of QHD, avoiding, however, the "meson-exchange" conception. This would enable us to avoid the controversy of the small distance description mentioned above. 
Another point is connected with the high densities. There are several interesting phenomena. The chiral phase transition and the disintegration of the nuclear matter to the quark-gluon plasma are much discussed nowadays. The possibility of the existence of the other phase states of nuclear matter containing the admixture of heavier baryons or of the "pion condensate" have been studied long ago [6, 7]. These effects can be important for the astrophysics. However the QHD parameters $g_{s, \omega}$ are defined at the saturation point. Hence, this approach cannot be expanded to the higher densities in a straightforward way. It is desirable to express the interactions in the scalar and vector channels through the observables whose density dependence can be found separately. This would enable to study the high density nuclear physics.

There are chances that both requirements can be realized. Recall that the QCD sum rules (SR) method invented by Shifman et al. [8] succeeded in expressing the static properties of the hadrons through the vacuum expectation values of several simplest operators of the quark and gluon fields (QCD operators of the lowest dimensions). If we succeed in expanding the SR approach for the case of finite densities, the in-medium modification of the values of the hadron parameters would be expressed through the in-medium values of the QCD condensates. Such approach would not require the conception of heavy meson $(\sigma$ and $\omega$ ) exchange. The calculation of the density dependence of QCD condensates would enable to use the approach in the broad interval of the density values.

Now I give a brief review of the SR method in vacuum, focusing on the points which we shall need at the finite values of density. There are several detailed reviews of the SR approach [9, 10].

\section{QCD sum rules in vacuum}

\subsection{Dispersion relations}

The basic point of SR method is the dispersion relation for the function $G\left(q^{2}\right)$ which describes the propagation of the system with the quantum numbers of a hadron. In the simplest form it is

$$
G\left(q^{2}\right)=\frac{1}{\pi} \int \frac{\operatorname{Im} G\left(k^{2}\right) d k^{2}}{k^{2}-q^{2}} .
$$


In quantum mechanics $G\left(q^{2}\right)$ is just the particle propagator. In the field theory different degrees of freedom are convenient in the different regions of the values of $q^{2}$. In particular, for the system with the baryon and electric charges equal to unity $Q=B=1$, the imaginary part $\operatorname{Im} G\left(k^{2}\right)=0$ at $k^{2}<$ $m^{2}$ with $m$ being the position of the lowest lying pole, i.e. $m$ is the proton mass. There are the other singularities at larger values of $k^{2}$. There are the cuts corresponding to the systems "proton+pions", etc. On the other hand, one can consider the system as that of three strongly interacting quarks. Such description becomes increasingly simple at $q^{2} \rightarrow-\infty$ due to the asymptotic freedom of QCD. This means that at $q^{2} \rightarrow-\infty$ the function $G\left(q^{2}\right)$ can be presented as the power series of $q^{-2}$ (and of QCD coupling constant $\alpha_{s}$ ). The coefficients of the expansion are the expectation values of local operators constructed of quark and gluon fields. These expectation values are called "condensates". Thus such presentation known as operator power expansion (OPE) [1], provides the perturbative expansion of the short-distance effects, while the nonperturbative physics is contained in the condensates.

Technically this means that one should start with the general presentation

$$
G\left(q^{2}\right)=i \int d^{4} x e^{i(q x)}\langle 0|T\{\eta(x) \bar{\eta}(0)\}| 0\rangle
$$

with $\eta$ being the local operator with the quantum numbers of the considered system. The operator $\eta(x)$ is the composition of the quark fields $\psi(x)$. For each quark field one can write in the lowest order of $\alpha_{s}$ (forgetting for a while about colours)

$$
\left\langle 0\left|T q_{\alpha}(x) \bar{q}_{\beta}(0)\right| 0\right\rangle=\frac{i}{2 \pi^{2}} \cdot \frac{\hat{x}_{\alpha \beta}+\frac{i m_{q}}{2} x^{2}}{x^{4}}-\frac{1}{4} \sum_{A} \Gamma_{\alpha \beta}^{X}\left\langle 0\left|: \bar{q}(0) \Gamma^{X} q(x):\right| 0\right\rangle
$$

with $\hat{x}=x_{\mu} \gamma^{\mu}$. This is the direct consequence of Wick theorem. In Eq. (3) $\alpha$ and $\beta$ are the Lorentz indices, $m_{q}$ stands for the quark mass. In the second term of the rhs of Eq. (3) $\Gamma^{X}$ is the complete set of the basic Dirac $4 \times 4$ matrices with the scalar, vector, pseudoscalar, pseudovector and tensor structures. The first term in the rhs of Eq. (3) is just the free propagator.

In the theories with the empty vacuum, e.g. in quantum electrodynamics the second term in rhs of Eq. (3) vanishes due to the normal ordering. In any field theory all the structures except the scalar one vanish due to the Lorentz invariance. In QCD the scalar term survives due to the spontaneous breaking of the chiral invariance. Thus

$$
\left\langle 0\left|T q_{\alpha}^{a}(x) \bar{q}_{\beta}^{b}(0)\right| 0\right\rangle=\frac{i}{2 \pi^{2}} \frac{\hat{x}_{\alpha \beta}+\left(i m_{q}\right) / 2 x^{2}}{x^{4}} \delta^{a b}-\frac{1}{12}\left\langle 0\left|\bar{q}^{a}(0) q^{a}(x)\right| 0\right\rangle
$$


with "a" and "b" standing for the colour indices. Note that Eq.(4) has a simple physical meaning. The quark can propagate between the space-time points "0" and " $x$ " as a free particle or by the exchange with the vacuum sea of the quark-antiquark pairs. Note, however, that Eqs. (3) and (4) are not presented in a gauge-invariant way (the operator $q(x)$ depends on the gauge of the gluon fields). The rigorous and gauge-invariant form of Eq. (4) is

$$
\left\langle 0\left|T q_{\alpha}^{a}(x) \bar{q}_{\beta}^{b}(0)\right| 0\right\rangle=\frac{i}{2 \pi^{2}} \frac{\hat{x}_{\alpha \beta}+i\left(m_{q}\right) / 2 x^{2}}{x^{4}} \delta_{a b}-\frac{1}{12}\left\langle 0\left|\bar{q}^{a}(0) q(0)\right| 0\right\rangle+0\left(x^{2}\right)
$$

with the expansion in powers of $x^{2}$ corresponding to the expansion of $G\left(q^{2}\right)$ in powers of $q^{-2}$.

To demonstrate the power of the dispersion relations I present, following [10], the derivation of the well-known Gell-Mann, Oakes-Renner relation (GMOR) 12

$$
\langle 0|\bar{u} u+\bar{d} d| 0\rangle=-\frac{2 f_{\pi}^{2} m_{\pi}^{2}}{m_{u}+m_{d}}
$$

with $f_{\pi}$ and $m_{\pi}$ being the decay constant and the mass of the pion. Recall that Eq. (6) is true in the chiral limit $m_{\pi}^{2} \rightarrow 0, m_{q} \rightarrow 0$.

The quantum numbers of pion can be carried by the axial current $A_{\mu}(x)=$ $\bar{u}(x) \gamma_{\mu} \gamma_{5} d(x)$ as well as by the pseudoscalar current $P(x)=i \bar{u}(x) \gamma_{5} d(x)$. Consider the dispersion relation for the function

$$
G\left(q^{2}\right)=i \frac{q^{\mu} \int d^{4} x e^{i(q x)}\left\langle 0\left|T A_{\mu}(x) \bar{P}(0)\right| 0\right\rangle}{q^{2}}
$$

using Eq. (5) for the quark propagators. The corresponding integral diverges at small $x$. Introducing a cutoff $x^{2} \geq L^{2}$ one finds in the limit $\alpha_{s}=0$

$$
G\left(q^{2}\right)=i\left(m_{u}+m_{d}\right) \frac{3}{8 \pi^{2}} \ln \frac{L^{2}}{-q^{2}}+i \frac{\langle 0|\bar{u} u+\bar{d} d| 0\rangle}{q^{2}} .
$$

To obtain the rhs of Eq. (1) recall about the partial conservation by axial current (PCAC) expressed by the equation $D^{\mu} A_{\mu}(x)=\sqrt{2} f_{\pi} m_{\pi}^{2} \varphi(x)$ with $\varphi$ standing for the pion field. Present

$$
\operatorname{Im} G\left(k^{2}\right)=\left\langle 0\left|A_{\mu} k^{\mu}\right| \pi(k)\right\rangle\langle\pi(k)|\bar{P}| 0\rangle \delta\left(k^{2}-m_{\pi}^{2}\right)+R\left(k^{2}\right)
$$

with the term $R\left(k^{2}\right)$ describing the higher lying states. Using PCAC one finds the term $R\left(k^{2}\right)$ to contain one more factor $m_{\pi}^{2}$ compared to the first 
term in rhs of Eq. (9). Hence, in the chiral limit we can neglect $R\left(k^{2}\right)$ as well as the first in rhs of Eq. (8). Thus the dispersion relation is

$$
i \frac{\langle 0|\bar{u} u+\bar{d} d| 0\rangle}{q^{2}}=\frac{\left\langle 0\left|A_{\mu} k^{\mu}\right| \pi\right\rangle\langle\pi|\bar{P}| 0\rangle}{m_{\pi}^{2}-q^{2}},
$$

with $k^{2}=m_{\pi}^{2}$. Calculating the matrix elements in the rhs by using PCAC and assuming $-q^{2} \gg m_{\pi}^{2}$ we come to Eq. (6).

This derivation of GMOR relation is complementary to the standard one presented in the QCD textbooks (see, e.g. [13]) which is based on physics of small momenta $q$. Usually GMOR is treated as the way to determine the value of $\langle 0|\bar{u} u+\bar{d} d| 0\rangle$. However in the framework of the developed approach it can be viewed as the relation which expresses the combination of the pion parameters $m_{\pi}^{2} f_{\pi}^{2}$ through the expectation value $\langle 0|\bar{u} u+\bar{d} d| 0\rangle$.

\subsection{Sum rules}

Unfortunately, the example considered above is the only case when the dispersion relation takes such a simple form. Usually there is no reason to neglect the higher lying physical states with respect to the lowest one. If the second lowest singularity is the cut starting at the point $W_{p h}^{2}$ we can present

$$
\operatorname{Im} G\left(k^{2}\right)=\lambda^{2} \delta\left(k^{2}-m^{2}\right)+f\left(k^{2}\right) \theta\left(k^{2}-W_{p h}^{2}\right)
$$

with $\lambda^{2}$ being the residue while $f\left(k^{2}\right)$ is the spectral function. Following previous discussion the lhs of Eq. (1) can be expanded in powers of $q^{-2}$ and thus Eq. (1) takes the form

$$
G_{O P E}\left(q^{2}\right)=\frac{\lambda^{2}}{m^{2}-q^{2}}+\frac{1}{\pi} \int_{W_{p h}^{2}} \frac{f\left(k^{2}\right) d k^{2}}{k^{2}-q^{2}}
$$

with the unknown parameters $m, \lambda^{2}$, and the unknown spectral function $f\left(k^{2}\right)$. The aim of the SR is to obtain the parameters of the lowest lying state. Hence, the second term of rhs of Eq. (12) is treated approximately. The approximation is prompted by the asymptotic behaviour

$$
f\left(k^{2}\right)=\frac{1}{2 i} \Delta G_{O P E}\left(k^{2}\right)
$$


at $k^{2} \gg\left|q^{2}\right|$. The integral over $k^{2} \gg\left|q^{2}\right|$ provides the terms $\sim \ln \left(L^{2} /-q^{2}\right)$ exceeding the contribution of the pole by this factor. The standard ansatz consists in extrapolation of Eq. (12) to the lower values of $k^{2}$, replacing also the physical threshold $W_{p h}^{2}$ by the unknown effective threshold $W^{2}$, i.e.

$$
\frac{1}{\pi} \int_{W_{p h}^{2}}^{\infty} \frac{f\left(k^{2}\right)}{k^{2}-q^{2}} d k^{2}=\frac{1}{2 \pi^{2} i} \int_{W^{2}}^{\infty} \frac{\Delta G_{O P E}\left(k^{2}\right)}{k^{2}-q^{2}} d k^{2},
$$

and thus the dispersion relation (1) takes the form

$$
G_{O P E}\left(q^{2}\right)=\frac{\tilde{\lambda}^{2}}{m^{2}-q^{2}}+\frac{1}{2 \pi^{2} i} \int_{W^{2}}^{\infty} \frac{\Delta G_{O P E}\left(k^{2}\right)}{k^{2}-q^{2}} d k^{2} .
$$

Such approximation of the spectrum is known as the "pole + continuum" model.

The lhs of Eq. (15) contains the QCD condensates. The rhs contains three unknown parameters $m, \lambda^{2}$ and $W^{2}$. However both lhs and rhs depend on $q^{2}$. The OPE becomes increasingly true at large values of $-q^{2}$. The "pole + continuum" model has sense only if the contribution of the continuum, treated approximately does not exceed the contribution of the pole, treated exactly. Thus the model becomes increasingly true at small values of $\left|q^{2}\right|$. The problem is to find the region of $\left|q^{2}\right|$ where both OPE and "pole + continuum" model are valid.

Such region is unlikely to exist in any channel of the dispersion relations presented by Eq. (15) with the necessary subtractions. To improve the overlap between the QCD and phenomenological descriptions Borel transform defined as

$$
\begin{array}{r}
B f\left(Q^{2}\right)=\lim _{Q^{2}, n \rightarrow \infty} \frac{\left(Q^{2}\right)^{n+1}}{n !}\left(\frac{-d}{d Q^{2}}\right)^{n} f\left(Q^{2}\right) \equiv \bar{f}\left(M^{2}\right) \\
Q^{2}=-q^{2} ; \quad M^{2}=Q^{2} / n
\end{array}
$$

was used in [8]. There are several useful features of the Borel transform. It removes the divergent terms in the lhs of Eq. (15) which are caused by the free quark loops - see, e.g., Eq. (8). This happens, since the Borel transform eliminates all the polynomials in $q^{2}$. Thus we do not need to make subtractions. Also it emphasizes the contribution of the lowest lying states 
in rhs of Eq. (15) due to the relation

$$
B \frac{1}{Q^{2}+m^{2}}=e^{-m^{2} / M^{2}} \text {. }
$$

The Borel transformed dispersion relations

$$
\tilde{G}_{O P E}\left(M^{2}\right)=\lambda^{2} e^{-m^{2} / M^{2}}+\frac{1}{2 \pi i} \int_{W^{2}}^{\infty} d k^{2} e^{-k^{2} / M^{2}} \cdot \Delta G_{O P E}\left(k^{2}\right)
$$

have been analyzed first for the vector mesons [8] and for the nucleons [14. In both cases the regions of the values of the Borel mass $M$ were found, where the matching of rhs and lhs of Eq. (18) was achieved. In particular, Ioffe [14] calculated the value of the meson mass as the function of QCD condensates. Several terms of OPE appeared to be needed to provide the matching of rhs and lhs of Eq. (18). The result of [14] can be treated as

$$
m=C\langle 0|\bar{u} u| 0\rangle
$$

with $C<0$ being the function of the gluon condensate and of the four-quark condensate $\langle 0|\bar{q} q \bar{q} q| 0\rangle$. The latter can be viewed as the expansion of the twoquark propagator, similar to Eq. (3). One can see that Eq. (19) has a simple physical meaning. The nucleon mass is caused by the quark exchange with the vacuum sea of $\bar{q} q$ pairs. The mechanism resembles that of the Nambu and Jona-Lasinio model.

The QCD SR method was applied successfully to calculation of the static properties of mesons [8] and nucleons [14, 15]. It provided new knowledge as well. For example, the value of gluon condensate $g_{0}=\left\langle 0\left|\frac{\alpha_{s}}{\pi} G_{\mu \nu} G_{\mu \nu}\right| 0\right\rangle$ was extracted by Vainshtein et al. [16] from the analysis of leptonic decays of $\rho$ and $\varphi$ mesons and from QCD analysis of charmonium spectrum. This condensate is a very important characteristics of QCD vacuum, since it is directly related to the vacuum energy density. The investigations based on the vacuum QCD sum rules are going on until now. The latest HEP preprint 17. which the value of $g_{0}$ has been calculated more accurately was published several months ago. 


\section{QCD sum rules in nuclear matter}

\subsection{The problems}

Now we discuss the possibility of the extension of QCD SR approach to the investigation of the characteristics of the nucleons in nuclear matter. If we succeed, the modification of the characteristics of the nucleon will be expressed through the expectation values of QCD operators in medium. Although some of the qualitative results may find the applications in the investigations of the finite nuclei, only the infinite nuclear matter will be considered below. In other words, the density of the distribution of the nucleons is the same in all the space points.

Since the Lorentz invariance is lost, the correlation function in medium

$$
G^{m}(q)=i \int d^{4} x e^{i(q x)}\langle M|T\{\eta(x) \bar{\eta}(0)\}| M\rangle
$$

depends on two variables, but not on $q^{2}$ only. The spectrum of the function $G^{m}(q)$ is much more complicated, than that of the vacuum correlator $G\left(q^{2}\right)$ defined by Eq. (2). The singularities can be connected with the nucleon (proton) placed into the matter, as well as with the matter itself. One of the problems is to find the proper variables, which would enable us to focus on the properties of our probe proton.

In the papers [18, 19] it was suggested to use $q^{2}$ as one of the variables. The shift of the position of the nucleon pole $m_{m}-m$ would be the unknown parameter to be determined from the SR equations. On the other hand

$$
m_{m}-m=U\left(1+0\left(\frac{U}{m}\right)\right)
$$

with $U$ being the single-particle energy of the nucleon. This is the very characteristics which enters the equation of state. To separate this singularity from the other ones a proper choice of the second variable is needed. Considering the nuclear matter as the system of $A$ nucleons with momenta $p_{i}$, introduce

$$
p=\frac{\Sigma p_{i}}{A}
$$

with $\mathbf{p}=0$ in the rest frame of the matter. Under the choice of $s=$ $(p+q)^{2}=$ const we avoid the singularities connected with the excitation of two nucleons [18]-[20]. The constant value of $s$ should be fixed by the 
condition that the probe proton is put on the Fermi surface of the matter. In the simplified case when the Fermi motion of the nucleons of the matter is neglected, we can just assume $s=4 m^{2}$.

Thus we shall write the dispersion relations for the function $G^{m}\left(q^{2}, s\right)-$ $G\left(q^{2}\right)$ with the functions $G$ and $G^{m}$ defined by Eqs. (2) and (20). It was shown in 21 that the nucleon pole is still the lowest lying singularity of the function $G^{m}\left(q^{2}, s\right)$ until we do not include the three-nucleon interactions. All the other singularities are lying at larger values of $q^{2}$ being quenched by the Borel transform due to Eq. (17). Thus we use the "pole + continuum" model for the spectrum of the function $G^{m}\left(q^{2}, s\right)$.

The OPE coefficients of $G^{m}\left(q^{2}, s\right)$ are the in-medium expectation values of QCD operators. Thus to use the SR equations one should find the density dependence of these condensates. It is not clear "a priori" if the OPE series converges indeed.

\subsection{Lowest order OPE terms}

In the lowest order of OPE the expectation values of the lowest dimension are involved only. In particular, there are the scalar expectation values

$$
\kappa^{i}(\rho)=\left\langle M\left|\bar{q}_{i} q_{i}\right| M\right\rangle
$$

with $q_{i}$ standing for " $u$ " or " $d$ " quark. There are also the vector expectation values

$$
v_{\mu}^{i}(\rho)=\left\langle M\left|\bar{q}_{i} \gamma_{\mu} q_{i}\right| M\right\rangle
$$

taking the form $v_{\mu}^{i}(\rho)=v^{i}(\rho) \delta_{\mu 0}$ in the rest frame of the matter. The condensates $\kappa$ have nonzero values in vacuum, while the vacuum values of the vector condensates vanish, i.e. $v_{i}(0)=0$. Due to the conservation of the vector current we find immediately

$$
v^{i}(\rho)=\frac{n_{i}^{(p)}+n_{i}^{(n)}}{2} \rho
$$

with $n_{i}^{p(n)}$ standing for the number of the valence quarks of the flavour " $i$ " in the proton (neutron).

The correlator $G^{m}\left(q^{2}\right)$ contains three structures being proportional to $\gamma_{\mu} p^{\mu}, \gamma_{\mu} q^{\mu}$, and $I$ with $I$ standing for the unit $4 \times 4$ matrix. Thus we obtain three QCD sum rules. There are four independent unknowns to be 
determined from SR equations. These are the three parameters of the nucleon which are the vector and scalar self-energies $\Sigma_{s}$ and $\Sigma_{s}$ and also the shift of the value of the residue $\lambda_{m}^{2}-\lambda^{2}$. The shift of the position of the pole can be expressed through the self-energies, i.e. $m_{m}-m=\Sigma_{v}+\Sigma_{s}$. One more unknown parameter is the shift of the position of the threshold $W_{m}^{2}-W^{2}$.

The explicit form of the SR equations is presented, e.g. in [21]. The matching of the rhs and lhs can be achieved in the same interval of the values of $M^{2}$ as in the case of vacuum. The shift of the position of the nucleon pole was found to be a superposition of the vector and scalar condensates [20]

$$
\begin{aligned}
& m_{m}=C_{1} \kappa(\rho)+C_{2} v(\rho) \\
& m_{m}-m=U(\rho)
\end{aligned}
$$

with the last equality coming form Eq. (21), $\kappa=\kappa^{u}+\kappa^{d}$. This provides the simple picture of formation of the value of $m_{m}$. Our probe proton exchanges quarks with the sea of $\bar{q} q$ pairs which differs from that in vacuum. This forms the Dirac effective mass $m^{*}=m+\Sigma_{s}$. This mechanism is described by the first term in rhs of Eq. (26). The exchange with the valence quarks adds the second term.

In the next to leading order of OPE the gluon condensate $g(\rho)=\left\langle M\left|\frac{\alpha_{s}}{\pi} G_{\mu \nu}\right| M\right\rangle$ should be taken into account. We shall see that numerically it is not very important.

\subsection{Gas approximation. The role of $\pi N$ sigma-term in nuclear physics}

We saw the lowest OPE to contain the condensates $v(\rho), \kappa(\rho)$ and $g(\rho)$. While the vector condensate is exactly linear in $\rho$, the condensates $\kappa(\rho)$ and $g(\rho)$ are more complicated functions of density. We start with the gas approximation in which the matter is treated as ideal Fermi gas of the nucleons. Thus our probe proton interacts with the system of non-interacting nucleons. In this approximation [18]

$$
\begin{aligned}
& \kappa(\rho)=\kappa(0)+\rho\langle N|\bar{q} q| N\rangle \\
& g(\rho)=g(0)+\rho\left\langle N\left|\frac{\alpha_{s}}{\pi} G_{\mu \nu} G_{\mu \nu}\right| N\right\rangle .
\end{aligned}
$$

The matrix elements in rhs of Eqs. (27) and (28) can be related to the 
observables. In particular,

$$
\langle N|\bar{q} q| N\rangle=\frac{2 \sigma}{m_{u}+m_{d}}
$$

with $\sigma$ being the pion-nucleon sigma term which is connected to the pionnucleon elastic scattering amplitude $T$ by the relation $T=-\sigma / f_{\pi}^{2}[22$. However in the latter relation $T$ denotes the amplitude in certain unphysical point. The experiments provide the data on the physical amplitude $T_{p h}=-\Sigma / f_{\pi}^{2}$ with $\Sigma=(60 \pm 7) \mathrm{MeV}$. The method of extrapolation of observable amplitude to the unphysical point was developed by Gasser et al. [23. They found

$$
\sigma=(45 \pm 7) \mathrm{MeV} \text {. }
$$

Note that from the point of chiral expansion, the difference $\Sigma-\sigma$ is of higher order, i.e. $(\Sigma-\sigma) / \sigma \sim m_{\pi}$.

Note also the physical meaning of the expectation value of the operator $\bar{q} q$ averaged over a hadron state. Anselmino and Forte [24, 25] showed that under reasonable model assumptions it can be treated as the total number of quarks and antiquarks.

As to gluon condensate, the expectation value is [26]

$$
\left\langle N\left|\frac{\alpha_{s}}{\pi} G_{\mu \nu} G_{\mu \nu}\right| N\right\rangle=-\frac{8}{9}\left(m-\Sigma m_{j} \cdot\left\langle N\left|q_{j} q_{j}\right| N\right\rangle\right)
$$

with $j$ standing for $u, d$ and $s$ quarks. This equation comes from the averaging of the QCD Hamiltonian over the nucleon state with the account of the additional relations found in [26. In the chiral limit only the strange quarks contribute. In the chiral SU(3) limit the second term in brackets in rhs of Eq. (31) turns to zero.

Solving the SR equations in the leading order of OPE in the gas approximation we find the potential energy to be [20]

$$
U(\rho)=\operatorname{bigg}[66 v(\rho)-32(\kappa(\rho)-\kappa(0))] \mathrm{GeV}^{-2}
$$

with the difference $\kappa(\rho)-\kappa(0)$ being described by the second term of the rhs of Eq. (27). Thus the potential energy is presented as the superposition of a positive term proportional to the vector condensate and a negative term proportional to the scalar condensate. At the saturation point $\rho=\rho_{0}=$ $0.17 \mathrm{fm}^{-3}=1.3 \cdot 10^{-3} \mathrm{GeV}^{3}$ we find the two terms in the rhs of Eq. (32) 
to be $200 \mathrm{MeV}$ and $-330 \mathrm{MeV}$. The gluon condensate adds about $10 \mathrm{MeV}$ to the vector term in the next to leading order of OPE Similar results were obtained in [27] in another SR approach based on the dispersion relations in $q_{0}$.

These are the common points between the SR approach and the Walecka model [20]. Note, however, that we did not need the fitting parameters like $g_{\omega}$ and $g_{\sigma}$ of QHD. The exchanges by the strongly correlated quarks (i.e. by the mesons) are expressed through the exchanges by the uncorrelated quarks. The interactions in the vector channel are calculated explicitly. The interactions in the scalar channel are expressed through the observable $\pi N$ sigma-term. Hence, in SR approach the $\sigma$-term determines the linear part of the scalar interactions.

\subsection{Beyond the gas approximation. A possible satura- tion mechanism}

Now we shall try to go beyond the gas approximation, remaining, however, in the lowest orders of OPE. Account of the interactions between the nucleons of the matter does not change Eq. (25) for the vector condensate. However the scalar condensate obtains additional contributions $S(\rho)$ caused by averaging of the operator $\bar{q} q$ over the meson cloud. Thus we have

$$
\kappa(\rho)=\kappa(0)+\rho\langle N|\bar{q} q| N\rangle+S(\rho)
$$

with a nonlinear behavior of $S(\rho)$ at small values of $\rho$.

Assume that the meson cloud consists of all kinds of the mesons $(\pi, \omega$, etc.). It was shown in [18] that in the chiral limit $m_{\pi}^{2} \rightarrow 0$ (neglecting also the finite size of the nucleons) one can obtain the function $S(\rho)$ as a power series in Fermi momenta $p_{F} \sim \rho^{1 / 3}$. The lowest order term $\sim \rho p_{F}$ comes from the one-pion Fock term (known also as the Pauli blocking term). The two-pion exchanges with the nucleons in the intermediate states contribute as $\rho p_{F}^{2}$ [28. The heavier mesons contribute as $\rho p_{F}^{3} \sim \rho^{2}$.

Even beyond the chiral limit we expect the pion cloud to provide the leading contribution to the nonlinear term $S(\rho)$. This is because the contributions of various mesons $X$ contains the meson expectation values $\langle X|\bar{q} q| X\rangle=n_{X}$ with $n_{X}$ being the total number of quarks and antiquarks. We can expect $n_{X} \approx 2$. However the pion expectation value calculated by the current al-

gebra technique [29] provides $\langle\pi|\bar{q} q| \pi\rangle=\frac{m_{\pi}}{m_{u}+m_{d}} \approx 12$. Thus the pion cloud contribution is enhanced. 
Note that the simplest account of the nonlinear terms in the scalar condensate signals the possible saturation mechanism. Assuming the chiral limit $m_{\pi}^{2}=0$ and including the Pauli blocking term only we can present $\kappa(\rho)=\kappa(0)+\frac{2 \Sigma}{m_{u}+m_{d}} \rho-3.2 \frac{p_{F}}{p_{F 0}} \rho[21]$ with $p_{F 0}=268 \mathrm{MeV} / \mathrm{c}$ being the Fermi momentum corresponding to the saturation value of density $\rho_{0}$. This provides the potential energy

$$
U(\rho)=\left[\left(198-42 \cdot \frac{2 \Sigma}{m_{u}+m_{d}}\right) \frac{\rho}{\rho_{0}}+133\left(\frac{\rho}{\rho_{0}}\right)^{4 / 3}\right] \mathrm{MeV},
$$

which contains the $\Sigma$-term and the pion-nucleon coupling constant $g_{\pi N N}$ as the only parameters. After adding the kinetic energy the energy functional obtains the minimum at $\rho=\rho_{0}$ if we put $\Sigma=62.8 \mathrm{MeV}(\sigma=47.8 \mathrm{MeV})$ which is consistent with the experimental data. The binding energy appears to be $\varepsilon=-9 \mathrm{MeV}$. The incompressibility coefficient which defines the shape of the saturation curve also has a reasonable value $K \approx 180 \mathrm{MeV}$.

Of course, the results for the saturation should not be taken too seriously. They are very sensitive to the exact value of the $\Sigma$ term. This is caused by the simplified model of the nonlinear effects. The rigorous treatment of the pion cloud requires the account of the multinucleon effects in the propagation of the pions [30, 31]. Inclusion of these effects [32, 33] still provides $S(\rho)<0$ at the densities close to the saturation value. Thus the nonlinear behaviour of the scalar condensate may be responsible for the saturation properties of the matter. However the results of this subsection can be considered only as the sign that further development of the approach may appear to be fruitful.

Thus the nonlinear behaviour of the scalar condensate is a possible source of the saturation. Here we find a certain analog of the QHD saturation mechanism. Recall that in Walecka model the saturation caused by a complicated dependence of the "scalar density" of the nucleons $\rho_{S}=\int \frac{d^{3} p}{(2 \pi)^{3}} \frac{m^{*}}{\varepsilon(p)} \theta\left(p_{F}-p\right)$ on the density $\rho=\int \frac{d^{3} p}{(2 \pi)^{3}} \theta\left(p_{F}-p\right)$.

Anyway, further development of the approach requires investigation of the higher order OPE terms as well as the analysis of the condensates beyond the gas approximation.

\subsection{Higher order OPE terms}

As we have seen, the vector and scalar condensates only contribute in the leading order of OPE. The gluon condensate contributes to the terms of the 
relative order $q^{-2}$ of the OPE of $G^{m}\left(q^{2}, s\right)$. We saw it to be numerically small, as well as some other contributions of this order which we did not discuss (see [33). However there are some problems with the terms of the order $q^{-4}$ which contain the four-quark condensates $h^{X Y}(\rho)=\left\langle M\left|\bar{q} \Gamma^{X} q \bar{q} \Gamma^{Y} q\right| M\right\rangle$.

The expectation values of the four-quark operators are not well established even in vacuum. The usual assumption is the factorization approximation [8] with the intermediate vacuum states dominating in all the channels. As to the in-medium values of the scalar condensate, one can separate the configuration with one of the products $\bar{q} q$ acting on vacuum states while the other one acts on the nucleon states [20]. Thus in the gas approximation

$$
h^{S S}(\rho)-h^{S S}(0)=2\langle 0|\bar{q} q| 0\rangle\langle N|\bar{q} q| N\rangle \rho+\left\langle N\left|(\bar{q} q \bar{q} q)_{\text {int }}\right| N\right\rangle \rho .
$$

In the second term of the rhs all the quark operators act inside the nucleon. Equations (6) and (29) enable us to find the magnitude of the first term of the rhs. If the first term estimates the values of all the four-quark condensates indeed, the term of the order $q^{-4}$ of OPE is numerically larger than the leading term at the values of the Borel mass where the SR equations are solved. This would cause doubts in the convergence of OPE. Fortunately the situation is not as bad as that. Celenza et al. 34 demonstrated that there is a strong cancellation between the two terms in rhs of Eq. (34). However the lack of information on the four-quark condensate have been an obstacle for the further development of the approach during many years. The recent calculations [35] are expected to improve the situation.

\subsection{New knowledge}

Independently of the magnitude of the contribution of the four-quark condensates, the SR predict certain new features of the nuclear forces.

\subsubsection{Anomalous structure of the nucleon-meson vertices}

As we have stated earlier, the QCD sum rules can be viewed as a connection between exchange of uncorrelated $\bar{q} q$ pairs between our probe nucleon and the matter and the exchange by strongly correlated pairs with the same quantum numbers (the mesons). In the conventional QHD picture this means that in the Dirac equation for the nucleon in the nuclear matter $(\hat{q}-\hat{V}) \psi=(m+\Phi) \psi$ the vector interaction $V$ corresponds to exchange by the vector mesons with the matter while the scalar interaction $\Phi$ is caused 
by the scalar mesons exchange. In the mean field approximation the vector interaction $V$ is proportional to density $\rho$, while the scalar interaction is proportional to the "scalar density" $\rho_{s}$ which is a more complicated function of the density $\rho$. Thus $V=V(\rho)$, while $\Phi=\Phi\left(\rho_{s}\right)$. We have seen that QCD sum rules provide similar picture in the lowest orders of OPE: vector and scalar parts $G_{v, s}$ of the correlator $G^{m}$ depend on vector and scalar condensates correspondingly: $G_{v}^{m}=G_{v}^{m}(v(\rho)) ; G_{s}^{m}=G_{s}^{m}(\kappa(\rho))$. However we find a somewhat more complicated dependence in the higher order OPE terms, say, $G_{s}^{m}=G_{s}^{m}(\kappa(\rho), v(\rho))$, depending on both scalar and vector condensates. This is due to the four-vector condensates. In particular, the scalar-vector condensate $\left\langle M\left|\bar{u} u \bar{d} \gamma_{0} d\right| M\right\rangle$ contains the contribution $\langle 0|\bar{u} u| 0\rangle\left\langle M\left|\bar{d} \gamma_{0} d\right| M\right\rangle$. This term is proportional to the vector condensate, contributing, however, to the SR for the scalar structure. In QHD picture this corresponds to the explicit dependence of the scalar interaction $\phi$ on the density $\rho$. Such dependence is not included in QHD, at least on the mean field level. These contributions correspond to the anomalous structure of vertex of the interaction between the nucleon and the scalar meson in the meson exchange picture. Similar situation takes place for the condensate $\langle 0|\bar{u} u| 0\rangle\langle M|\bar{u} u| M\rangle$ contributing to the SR for the vector structure.

\subsubsection{Charge-symmetry breaking forces}

There is an old problem of the difference between the strong interactions of the proton and neutron with the systems containing equal numbers of protons and neutrons [36]. In framework of SR method the difference can be caused by the explicit dependence of the function $G^{m}$ on the current quark masses and on the isospin breaking expectation value $\gamma=\frac{\langle M|\bar{d} d-\bar{u} u| M\rangle}{\langle M|\bar{u} u| M\rangle}$. The problem was attacked by the SR approach in a number of works [37. The proton-neutron binding energy difference was expressed through the quark mass difference $m_{d}-m_{u}$ and the condensate $\gamma$ under various additional assumptions. The SR analysis provided some qualitative results which may be useful in the building of the charge-symmetry breaking nuclear forces (CSB). One of them is the importance of CSB in the scalar channel. Earlier there was a common belief that the CSB in the vector channel are responsible for the effect. Another point is that the mixed structures described in Subsec. 3.6.1, manifest themselves in the leading terms of OPE. Thus explicit dependence of vector and scalar forces on both $\rho$ and $\rho_{s}$ may become important. 


\subsection{A self-consistent scenario [32,33]}

A rigorous calculation of the contribution of the pion cloud to the scalar condensate $\kappa(\rho)$ requires the consistent treatment of the pion dynamics in nuclear matter. The nonlinear term $S(\rho)$ can be presented as

$$
S(\rho)=\frac{2 m_{\pi}^{2}}{m_{u}+m_{d}} \frac{\partial \Sigma_{\pi}(\rho)}{\partial m_{\pi}^{2}}
$$

with $\Sigma_{\pi}$ being the pion contribution to the nucleon self-energy. This can be obtained by using the presentation $S=d V / d m_{q}$ (with $V$ standing for the interaction energy) obtained by Cohen et al. 38. and taking into account the pion contribution only. The self-energy $\Sigma_{\pi}$ contains the in-medium pion propagator $D$ which satisfies the Dyson equation

$$
D=D_{0}+D_{0} \Pi D \text {. }
$$

The propagator $D$ differs from the free propagator $D_{0}$ due to the particlehole excitations described by the polarization operator $\Pi$. The latter can be expressed through the amplitude of the forward $\pi N$ scattering (this provides the value $\left.\Pi_{0}\right)$. The short-range correlations can be described by means of the Finite Fermi System Theory (FFST) introduced by Migdal [30]. If only the nucleon-hole excitations are included, the operator $\Pi_{0}$ turns to $\Pi=\Pi_{0} /(1+$ $\left.g_{N} \Pi_{0}\right)$ with $g_{N}$ being a FFST constant determined from the experimental data. The account of long-range correlations [39] modifies the effective value of $g_{N}$.

The function $S(\rho)$ thus depends on the effective mass of the nucleon $m^{*}(\rho)$ and on the $\pi N$ coupling constant $g_{\pi N N}^{*}(\rho)$. The latter can be presented through the fundamental parameters $f_{\pi}$ and axial coupling constant $g_{A}$ by Goldberger-Treiman relation 40.

$$
\frac{g_{\pi N N}}{2 m}=\frac{g_{A}}{2 f_{\pi}},
$$

which can be expanded to the case of the finite density.

On the other hand, the SR provide the dependence of the nucleon effective mass on the condensate $\kappa(\rho)$. If we succeed in describing the dependence of $g_{A}(\rho, \kappa(\rho))$ (the first steps were made in [20]) and of $f_{\pi}(\kappa(\rho), \rho)$, we shall come to the set of self-consistent equations

$$
m^{*}=m^{*}(\kappa(\rho)) ; \quad f_{\pi}^{*}=f_{\pi}^{*}(\kappa(\rho)) ; \quad g_{A}=g_{A}^{*}(\kappa(\rho)) ; \quad \kappa=\kappa\left(m^{*}(\rho), \frac{g_{A}^{*}(\rho)}{f_{\pi}^{*}(\rho)}\right) .
$$


The first three equations should originate from the sum rules. The last one is just the combination of Eqs. (33), (36) and (38). One should add similar equations for the delta-isobar.

This would enable to describe the baryon parameters in the hadron phase of the baryon matter up to the point of the chiral phase transition where $\kappa(\rho)=0$.

\subsection{A sub-plot: Goldstone pions never condense [32,33,41,42]}

The possibility of the "pion condensation" was first discussed by Migdal [7. The observation is that at certain value of density $\rho=\rho_{\pi}$ the pion propagator presented by Eq. (36) obtains the pole at the energy $\varepsilon_{\pi}=0$. This would signal the degeneracy of the ground state of the system. The ground state contains an admixture of the oscillations with the quantum numbers of the pions. The value of $\rho_{\pi}$ appeared to be sensitive to the values of FFST constants, being $\rho_{\pi} \geq 2 \rho_{0}$ in most of the hadronic models.

The analysis shows that the singularity of the pion propagator in the point of the "pion condensation" leads to the divergence of the function $S(\rho)$ expressed by Eq. (35). This provides $\kappa(\rho) \rightarrow+\infty$ at $\rho \rightarrow \rho_{\pi}$, while the density increases. On the other hand, $\kappa(0)<0$. Thus $\kappa=0$ at certain point $\rho_{c h}$ between the zero value and $\rho_{\pi}$, i.e. $0<\rho_{c h}<\rho_{\pi}$. However, since $\kappa\left(\rho_{c h}\right)=0$, the chiral symmetry is restored at $\rho=\rho_{c h}$. One cannot expand the hadronic physics of the densities close to the saturation value $\rho_{0}$ to the region $\rho \geq \rho_{c h}$. Thus the "pion condensation" point cannot be reached in framework of the existing hadronic models. Anyway, once the chiral symmetry is restored, the pions do not exist as the Goldstone bosons any more. Thus, there is no "pion condensation" of the Goldstone pions.

The subject is analyzed in details in the papers [41, 42]. Although not being connected with the SR directly, this result is the outcome of investigation of the scalar condensate stimulated by studies of the sum rules.

\section{Summary}

We saw that the first steps in the application of QCD sum rules method to investigation of the in-medium nucleon parameters are successful. The effective mass and the single-particle potential energy where expressed through QCD condensates in the lowest OPE orders. The quark and gluon condensates 
are expressed through the observables. The pion-nucleon $\sigma$-term appeared to determine the value of the scalar forces. The approach reproduced one of the key points of QHD picture: the nucleon moves in superposition of scalar and vector fields which cancel to large extent. The numerical values are consistent with those of QHD. However the approach does not use the fitting parameters and avoids the controversial conception of the heavy meson exchange by the point nucleons.

The approach provides also some new knowledge about the nuclear forces. These are the anomalous structures of the meson-nucleon vertices. Such contributions usually are not included in QHD. Another point is the importance of the scalar channel in the charge-symmetry breaking forces.

Even the simplified model for the in-medium scalar condensate provides a possible mechanism of saturation. It is caused by the nonlinear contribution of the pion cloud to this expectation value. In the rigorous treatment of the pion dynamics the sum rule for the effective mass of the nucleon $m^{*}$ and the expression for the pion contribution to the condensate form the set of self-consistent equations. The complete set of the equations should include the sum rules for the axial coupling constant $g_{A}^{*}$ in medium and for the inmedium pion decay constant $f_{\pi}^{*}$. Investigation of the complete set of the equations is the subject of the future work.

The development of the method requires calculation of the troublesome four-quark condensates. The calculation of complete set of these expectation values in framework of the convincing models is in progress.

I thank M. G. Ryskin, V. A. Sadovnikova and E. M. Levin for fruitful cooperation during many years. I am indebted to V.M. Braun, G.E. Brown, E.M. Henley, B.L. Ioffe, L. Kisslinger, M. Rho, E.E. Saperstein and C.M. Shakin for numerous discussions. The work was supported in part by Deutsche Forschungsgemeinschaft (DFG) — grant 438/RUS113/595/0-1 and by Russian Foundation for Basic Research (RFBR) — grants 0015096610 and 0002 16853. 


\section{References}

[1] J.D. Walecka, Ann. Phys. 83 (1974) 491.

[2] L.S. Celenza and C.M. Shakin, "Relativistic nuclear physics" (World Scientific, Philadelphoa, 1986).

[3] B.D. Serot and J.D. Walecka, Adv. Nucl. Phys. 16 (1985) 1.

[4] J.W. Negele, Comm. Nucl. Part. Phys. 14 (1985) 303.

[5] L.A. Sliv, M.I. Strikman and L.L. Frankfurt, Sov. Phys. - Uspekhi 28 (1985) 281.

[6] J. Boguta, Phys. Lett. B 109 (1982) 251.

[7] A.B. Migdal, Sov. Phys. JETP 34 (1972) 1184.

[8] M.A. Shifman, A.I. Vainshtein and V.I. Zakharov, Nucl. Phys. B 147 (1979) 385, 448, 519.

[9] M.A. Shifman, "Vacuum structure and QCD sum rules" (North Holland, Amsterdam, 1992).

[10] L.J. Reinders, H. Rubinstein, S. Yazaki, Phys. Rep. 127 (1985) 1.

[11] K.G. Wilson, Phys. Rev. 179 (1969) 1499.

[12] M. Gell-Mann, R.J. Oakes and B. Renner, Phys. Rev. 175 (1968) 2195.

[13] F.J. Yndurain, "Quantum chromodynamics" (Springer-Verlag, NY, 1983).

[14] B.L. Ioffe, Nucl. Phys. B 188 (1981) 317.

[15] B.L. Ioffe and A.V. Smilga, Nucl. Phys. B 232 (1984) 109;

V.M. Belyaev and Y.I. Kogan, Sov. Phys. JETP Lett. 37 (1983) 730.

[16] A.I. Vainshtein, V.I. Zakharov and M.A. Shifman, JETP Lett. 27 (1978) 55.

[17] B.L. Ioffe and K.N. Zyablyuk, hep-ph/0207183. 
[18] E.G. Drukarev and E.M. Levin, JETP Lett. 48 (1988) 338; Sov. Phys. JETP 68 (2989) 680.

[19] E.G. Drukarev and E.M. Levin, Nucl. Phys. A 511 (1990) 679.

[20] E.G. Drukarev and E.M. Levin, Prog. Part. Nucl. Phys. 27 (1991) 77.

[21] E.G. Drukarev and M.G. Ryskin, Nucl. Phys. A 578 (1994) 333.

[22] T.P. Cheng and R. Dashen, Phys. Rev. Lett. 26 (1971) 594.

[23] J. Gasser, M.E. Sainio and A. Švarc, Nucl. Phys. B 307 (1988) 779;

J. Gasser, H. Leutwyler and M.E. Sainio, Phys. Lett. B 253 (1991) 252, 260.

[24] M. Anselmino and S. Forte, Z. Phys. C 61 (1994) 453.

[25] S. Forte, Phys. Rev. D 47 (1993) 1842.

[26] M.A. Shifman, A.I. Vainshtein and V.I. Zakharov, Phys. Lett. B 78 (1978) 443.

[27] R.J. Furnstahl, D.K. Grigel, and T.D. Cohen, Phys. Rev. C 6 (1992) 1507.

[28] E.G. Drukarev, M.G. Ryskin, and V.A. Sadovnikova, Z. Phys. A 353 (1996) 455.

[29] J. Gasser, Ann. Phys. 136 (1981) 62.

[30] A.B. Migdal, "Theory of finite Fermi systems and applications to atomic nuclei", Willey, New York, 1967.

[31] T. Ericson and W. Weise, "Pions and nuclei" (Clarendon Press, Oxford, 1988).

[32] E.G. Drukarev, M.G. Ryskin, and V.A. Sadovnikova, Eur. Phys. J. A 4 (1999) 171.

[33] E.G. Drukarev, M.G. Ryskin, and V.A. Sadovnikova, Prog. Part. Nucl. Phys. 47 (2001) 73. 
[34] L.S. Celenza, C.M. Shakin, W.D. Sun, and J. Szweda, Phys. Rev. C 51 (1995) 937.

[35] E.G. Drukarev, M.G. Ryskin, V.A. Sadovnikova, V.E. Lyubovitskij, T. Gutsche, and A. Faessler, - to be published.

[36] G.A. Miller, B.M.K. Nefkens, and I. Slaus, Phys. Rep. 194 (1990) 1.

[37] T. Hatsuda, H. Hogaasen, and M. Prakash, Phys. Rev. C 42 (1990) 2212; C. Adami and G.E. Brown, Z. Phys. A 340 (1991) 93;

T. Schafer, V. Koch, and G.E. Brown, Nucl. Phys. A 562 (1993) 644; E.G. Drukarev and M.G. Ryskin, Nucl. Phys. A 572 (1994) 560; A 577 (1994) 375c.

[38] T.D. Cohen, R.J. Furnstahl, and D.K.Griegel, Phys. Rev. C 45 (1992) 1881.

[39] W.H. Dickhoff, A. Faessler, H. Muther, and S.-S. Wu, Nucl. Phys. A 405 (1983) 534;

W.H. Dickhoff, A. Faessler, J. Meyer-ter-Vehn, and H. Muther, Phys. Rev. C 23 (1981) 1154.

[40] M.L. Goldberger and S.B. Treiman, Phys. Rev. 110 (1958) 1478.

[41] V.A. Sadovnikova and M.G. Ryskin, Phys. Atom. Nucl. 64 (2002) 440.

[42] V.A. Sadovnikova, preprints nucl-th/0001025; nucl-th/0201023. 